\title{
Philosophiques
}

\section{L'usage de la conjecture technique chez Galien de Pergame}

\section{Jérémie Hébrard}

Volume 46, numéro 1, printemps 2019

URI : https://id.erudit.org/iderudit/1062018ar

DOI : https://doi.org/10.7202/1062018ar

Aller au sommaire du numéro

Éditeur(s)

Société de philosophie du Québec

ISSN

0316-2923 (imprimé)

1492-1391 (numérique)

Découvrir la revue

Citer cet article

Hébrard, J. (2019). L'usage de la conjecture technique chez Galien de Pergame. Philosophiques, 46(1), 179-206. https://doi.org/10.7202/1062018ar

\section{Résumé de l'article}

L'enjeu de cette contribution est d'explorer les réflexions sur les arts stochastiques dans l'Antiquité et de montrer comment les développements concernant « l'art de conjecturer » que l'on trouve chez Galien constituent une option préférable aux voies traditionnelles empruntées par les philosophes pour expliquer la faillibilité inhérente à l'art médical. En distinguant le diagnostic scientifique du diagnostic conjectural, Galien englobe la totalité des cas pertinents pour l'art médical. Le premier, en raison de son caractère général, peut être théorisé. Quant au second, il concerne uniquement le particulier et n'est donc pas susceptible d'entrer dans la théorie, si ce n'est que par la méthode qui le rend possible. Il s'agit donc de dresser une ébauche des mécanismes qui sous-tendent la conjecture technique afin d'exposer la position adroitement élaborée par Galien sur un sujet où l'hégémonie philosophique régnait jusqu'alors. 


\title{
L'usage de la conjecture technique chez Galien de Pergame
}

\author{
JÉRÉMIE HÉBRARD \\ Université de Sherbrooke \\ jeremie.hebrard@Usherbrooke.ca
}

\begin{abstract}
RÉSUMÉ. - L'enjeu de cette contribution est d'explorer les réflexions sur les arts stochastiques dans l'Antiquité et de montrer comment les développements concernant «l'art de conjecturer» que l'on trouve chez Galien constituent une option préférable aux voies traditionnelles empruntées par les philosophes pour expliquer la faillibilité inhérente à l'art médical. En distinguant le diagnostic scientifique du diagnostic conjectural, Galien englobe la totalité des cas pertinents pour l'art médical. Le premier, en raison de son caractère général, peut être théorisé. Quant au second, il concerne uniquement le particulier et n'est donc pas susceptible d'entrer dans la théorie, si ce n'est que par la méthode qui le rend possible. II s'agit donc de dresser une ébauche des mécanismes qui sous-tendent la conjecture technique afin d'exposer la position adroitement élaborée par Galien sur un sujet où l'hégémonie philosophique régnait jusqu'alors.
\end{abstract}

\begin{abstract}
The aim of this paper is to examine the considerations on stochastic arts in Antiquity and to show how Galen's analysis concerning the "art of conjecturing" constitutes a preferable alternative to the traditional ways used by philosophers to explain the inherent fallibility in the medical art. By distinguishing the scientific diagnosis from the conjectural one, Galen encompasses all cases relevant to the medical art. The former, because of its general nature, can be theorized. As for the latter, it concerns only the individual and is therefore not likely to enter the theory, except by the method that makes it possible. This paper attempts to outline the modus operandi underlying the technical conjecture in order to illustrate Galen's deftly developed position on a subject almost exclusively investigated by philosophers until then.
\end{abstract}

\section{Introduction}

Galien est sans doute possible l'un des auteurs les plus méconnus de l'Antiquité, et ce, malgré (voire en raison de) l'ampleur du corpus qu'il a laissé derrière lui. Considéré comme le second plus grand médecin de toute l'Antiquité, après Hippocrate, il représente une source de savoir incontournable et intarissable. Pourtant, une certaine hésitation demeure lorsqu'on évoque le nom du médecin de Pergame. En tâtant le pouls des philosophes, on s'aperçoit que cette perplexité semble être causée par un simple manque de familiarité avec l'œuvre de Galien, encore que cela n'ait rien d'étonnant. D'une part, l'immensité du corpus galénique a de quoi intimider quiconque voudrait entreprendre une étude complète de Galien, d'autre part, et ceci découle certainement de cela, il y a une absence criante d'édi- 
tions critiques des textes originaux et un nombre assez restreint d'œuvres traduites ${ }^{1}$.

À la question: ne devrions-nous pas l'étudier en qualité de médecin plutôt qu'en qualité de philosophe, nous n'apporterons qu'une réponse indirecte. D'autres avant nous s'y sont frottés de manière plus directe, notamment Micheal Frede, qui a participé au renouveau des études philosophiques sur Galien. Dans le chapitre On Galen's Epistemology, Frede a montré que l'intérêt de Galien pour la philosophie, loin d'être ornemental, constitue un pilier soutenant l'édifice de sa réflexion sur la médecine. Évidemment, Galien laisse de côté des sujets philosophiques essentiels qu'il ne croit pas utile d'aborder puisqu'ils sont sans conséquence pour la médecine. À ce propos, répétons qu'il était avant tout médecin, et ses réflexions sur l'éthique ou la physique sont généralement orientées vers la pratique de l'art médical. Toutefois, dire que Galien était un médecin n'est pas incompatible avec l'affirmation qu'il avait pensé à sa pratique en tant que philosophe, à tout le moins pour s'expliquer sur les fondements de sa technique. Comme le nom de Galien est mentionné à de nombreuses reprises dans les œuvres d'Alexandre d'Aphrodise, de Thémistocle, de Simplicius et de Philopon, il est possible, à la Wittgenstein, de parler d'un «air de famille» entre eux ${ }^{2}$. Galien ferait ainsi partie de la famille des philosophes, même s'il en est un membre relativement éloigné.

En guise de contribution à l'étude de Galien philosophe, nous poursuivons deux objectifs en travaillant sur la stochastique. Au premier plan, il s'agira de donner un aperçu de la réflexion des philosophes (Platon, Aristote, Alexandre d'Aphrodise) et des médecins (Hippocrate, Galien) sur l'incertitude et la conjecture dans les techniques en général, et en médecine en particulier. Au second plan, il s'agira d'une analyse de l'aspect stochastique de la médecine chez Galien visant à convaincre le lecteur que certaines réflexions du médecin de Pergame n'ont rien à envier à celles des philosophes sur la même question.

\section{Le double aspect stochastique de la médecine chez Galien}

Avant tout, nous fournirons une définition sommaire de ce en quoi consiste un "art stochastique». Dans le traité d'un pseudo-Galien intitulé Le médecin. Introduction, on peut lire:

[...] on distingue deux sortes d'arts: les uns atteignent toujours la fin qui leur est conforme, comme la menuiserie, la construction navale, l'architecture; les

1. Nous ne pouvons qu'admirer l'initiative de Jacques Jouanna et Véronique BoudonMillot qui ont entrepris la traduction des œuvres de Galien dans la C.U.F. aux éditions Les Belles Lettres. À ce propos, et pour de plus de renseignements à propos des éditions précédentes du Corpus de Galien, consulter l'article de J. Jouanna et V. Boudon-Millot «Présentation du projet d'édition de Galien dans la collection des Universités de France», I993, p. IOI-I35.

2. Frede, I98I, p. 66. 
autres visent leur fin, comme on vise un but, et ne la trouvent pas toujours, mais la plupart du temps ${ }^{3}$, c'est pourquoi on les dit aussi conjecturaux ( $\sigma \tau о \chi \alpha \sigma \tau 1 \kappa \alpha i)$; parmi eux se trouverait la médecine, avec la rhétorique, l'art de piloter, et le tir à l'arc ${ }^{4}$.

Ce problème, que nous désignerons par la formule: "faillibilité des arts stochastiques", a occupé de nombreux philosophes anciens. Ces derniers, s'ils voulaient préserver le statut de $\tau \dot{\chi} \chi v \eta$ des arts stochastiques, devaient rendre compte de leur faillibilité tout en montrant que les échecs ne provenaient pas nécessairement de l'incapacité de l'artisan. Dans l'éventualité où l'on serait incapable d'expliquer pourquoi certaines tentatives se résolvent par un échec malgré la bonne conduite du traitement de la part du praticien, ce dernier semblera incompétent aux yeux du profane. Qui plus est, non seulement ses échecs seront attribués à son incompétence, mais ses réussites pourraient tout aussi bien être imparties à sa chance. Le cas échéant, il deviendrait simplement absurde d'affirmer que le physicien, le barreur ou le rhéteur possèdent réellement un savoir, leur succès étant dû au hasard ${ }^{5}$. Nous verrons donc brièvement le traitement réservé aux arts stochastiques chez Hippocrate, Platon et Aristote ${ }^{6}$. Ce survol nous permettra aussi d'apprécier plus justement les approfondissements apportés par Alexandre d'Aphrodise et Galien.

Il y a toutefois un second sens propre à Galien en vertu duquel on peut affirmer que la médecine est un art stochastique. Il s'agit de l'importance qu'il accorde à l'usage de la conjecture. Plus particulièrement, on trouve chez Galien des approfondissements concernant un "art de conjecturer" ${ }^{7}$. Cet art est toutefois l'apanage des médecins les plus expérimentés. Comme

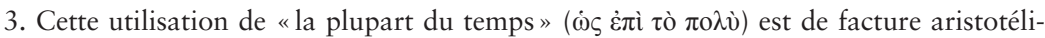
cienne plutôt que galénique.

4. Galien. Le médecin. Introduction, Kühn (XIV, 685, L.11 - L.15), traduction de C. Petit, 2009, p. II.

5. La question de l'irrégularité des résultats en médecine est l'objet du traité De l'Art du Corpus hippocratique. L'auteur entreprend de défendre l'art médical contre ceux qui chercher à miner sa crédibilité en affirmant que (I) les guérisons sont dues à la chance, (2) qu'il arrive que les patients guérissent sans l'intervention du médecin, (3) que certains patients meurent malgré l'intervention du médecin, (4) ou encore que les médecins refusent de traiter des cas devant lesquels ils sont impuissants. Sur ce traité, voir l'introduction du The Art de W. H. S. Jones (I923) dans Loeb Classical Library I48, p. I 86.

6. Cette démarche a déjà été entreprise à plusieurs reprises par nos contemporains. Toutefois, ces analyses nous semblent souvent fragmentaires et aucune synthèse réunissant tous ces auteurs à la fois n'a été entreprise. Aussi croyons-nous qu'il soit nécessaire de poursuivre dans cette voie, au risque de répéter ce que certains ont déjà dit.

7. À l'exception de cette occasion, nous utiliserons plutôt les termes de «conjecture technique ", suivant ainsi la traduction française de Véronique Boudon-Millot (2003). Cette

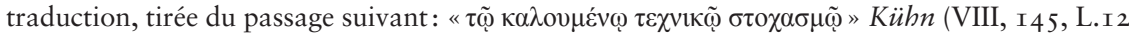
- L.I3), suggère par ailleurs que l'expression grec n'allait pas déjà de soi. Ce curieux alliage nous semble d'ailleurs encore en deçà de l'oxymore provoqué par l'idée qu'il soit possible de développer un «art de conjecturer». 
il l'écrit dans son Commentaire sur le régime des maladies aigües d'Hippocrate: "Making technical conjectures is proper to that man who has learned

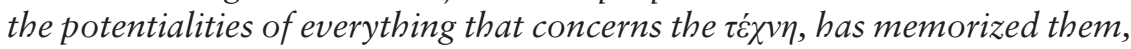
and industriously practised them ${ }^{8}$.» Pour l'instant, nous nous contenterons d'indiquer que l'usage de la conjecture chez Galien est double. Premièrement, la pratique de l'art médical requiert fréquemment d'établir des diagnostics et des pronostics précis qui faciliteront le choix de la thérapie. Il n'est pourtant pas rare que cela soit impraticable et que le médecin ait à déterminer la source de l'affection en usant de conjectures. Deuxièmement, le choix final de la thérapie ainsi que le dosage des remèdes nécessitent parfois d'y recourir' ${ }^{9}$. La plupart des études galéniques concernant la conjecture se penchent avant tout sur son rôle au sein de l'art médical. Aussi notre connaissance concernant leur modus operandi demeure-t-elle lacunaire. Notre objectif sera de dresser une ébauche des modalités qui sous-tendent l'utilisation de la conjecture technique chez Galien.

\section{1. $\sigma \tau 0 \chi-a ́ \zeta o \mu \alpha \iota$ dans la philosophie antique}

Le Liddell-Scott-Jones distingue deux significations pour le terme «Stokazomai» et ses déclinaisons. La première: [to] aim or shoot at - endeavour to make at - guess at a thing, est d'usage courant, comme nous le verrons à l'instant. Quant à la seconde, plus rare, elle désigne l'action de conjecturer: one must form a conjecture - proceeding by guesswork ${ }^{10}$. Nous passerons en revue les réflexions des prédécesseurs de Galien afin de montrer que l'usage qu'il fait du terme s'en démarque et donne un caractère unique à son développement sur les arts stochastiques.

\section{Hippocrate}

La thèse selon laquelle la médecine était déjà reconnue comme un art stochastique chez Hippocrate a été soutenue par Ingenkamp. Selon lui, les affirmations contenues dans le traité De l'ancienne médecine concernant la nécessité d'estimer les dosages des drogues permettaient de conclure que la réflexion sur l'aspect stochastique de la médecine tirait sa source du Corpus hippocratique ${ }^{11}$. Plus récemment, Boudon-Millot a soutenu que l'usage du

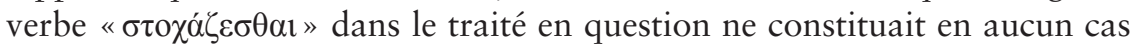
une preuve suffisante, le terme ne signifiant rien de plus que de "viser une certaine mesure» dans les cas où le médecin doit estimer avec précision la

8. Galien, In Hippocratis de victu acutorum commentaria, Kühn (XV, 585, L.7 - L.9)

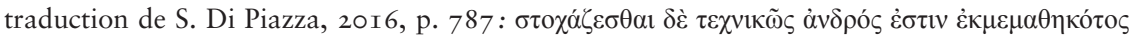

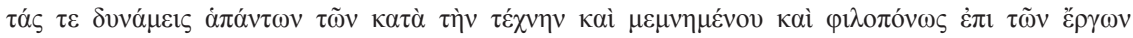
$\tau \varepsilon \tau \rho \mu \mu \varepsilon ́ v o v$.

9. Allens, I994, p. I00.

10. Henry George Liddell, et al., I996, p. I650.

11. Voir l'article de Heinz Gerd Ingenkamp, I983. 
quantité de nourriture à donner à un patient ${ }^{12}$. Mann semble aussi corroborer cette hypothèse. Selon lui, les affirmations dans De l'ancienne médecine ne signifient pas que le médecin doit user de conjecture, mais plutôt qu'il doit chercher à viser une mesure entre deux extrêmes, à savoir l'excès et le manque ${ }^{13}$. En ce sens, le médecin hippocratique n'use pas de conjectures. Il procède en ayant pour objectif d'apporter un équilibre entre les humeurs et vise $(\sigma \tau 0 \chi \alpha ́ \zeta \varepsilon \sigma \theta \alpha \mathrm{l})$ à se rapprocher de son but avec le plus de justesse possible. Nous partageons donc la conclusion de Boudon-Millot selon laquelle, même si tous les éléments sont en place dans le Corpus hippocratique pour élaborer une réflexion sur la médecine en tant qu'art stochastique, cela n'est jamais vraiment réalisé à nos yeux de contemporains.

\section{Platon}

L'attitude de Platon à l'égard de la médecine et du rapport qu'elle entretient avec la stochastique est plus partagée. Dans le Gorgias, Socrate oppose les arts qui ont soin du corps et de l'âme à leur contrefaçon qui use de la flatterie pour se donner la parure d'un art. La médecine est opposée à la cuisine alors que la justice est opposée à la rhétorique. Cette division est fondée sur le rapport que les arts authentiques entretiennent avec leur objet. Elles ont toujours pour but le meilleur état de la chose auxquelles elles se rapportent, alors que leurs contrefaçons «cherchent à atteindre le plaisir ${ }^{14}$ ». La cuisine, contrairement à la médecine, est en effet incapable de fournir une explication des raisons pour lesquelles elle obtient du succès. Cette bipartition des arts visant le bien de leur objet contre ceux qui visent le plaisir fournit à

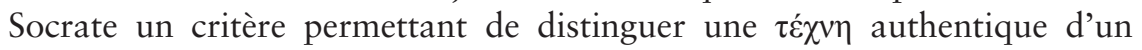
simple savoir-faire reposant sur l'expérience ${ }^{15}$. Cette attitude favorable à l'égard de la médecine se retrouve aussi dans le Phèdre, où Socrate recommande aux orateurs d'appliquer la méthode d'enquête d'Hippocrate à l'étude de l'âme, sans quoi la rhétorique est condamnée à demeurer une démarche d'aveugle qui procède à tâtons (Phèdre 270c-d $)^{16}$.

Le sentiment de Platon envers la médecine semble s'être modifié plus tard dans sa vie, particulièrement dans le Philèbe. Cette fois, l'art médical est rangé au côté des arts conjecturaux comme la musique et la navigation, qui sont imprécises et peu assurées. À celles-ci, Platon oppose les arts qui

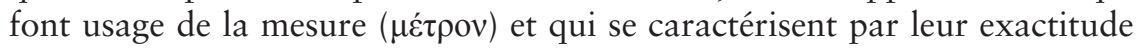

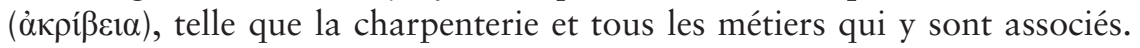
Autrement dit, dans cette nouvelle bipartition, Platon divise les arts selon

12. Véronique Boudon-Millot, 2005, p. 92.

13. Joel E. Mann, 2008, p. II 2.

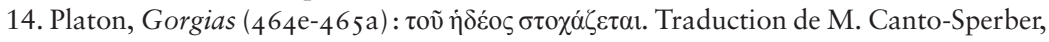
2OII, p.435

15. Robert Jim Hankinson, 2004, p. 4.

16. Platon, Phèdre (270c-d, traduction de L. Brisson, M. Canto-Sperber, 20 I I, p. I 287. 
qu'ils font usage de la mathématique ou de la conjecture et de l'approximation ${ }^{17}$. Qualifiés de peu sûrs, les seconds possèdent un statut inférieur, sans

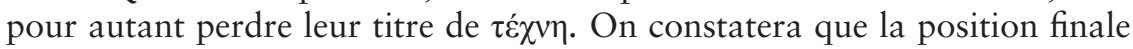
de Platon quant aux arts conjecturaux est très contraignante: "Plato requires the highest degree of accuracy, there is no middle way, there is no alternative between gignoskein and stochazestai, everything without akribeia $[. .$.$] falls within the condemnable area of conjecture { }^{18}$.»

\section{Aristote}

La rigidité de Platon sera d'ailleurs la cible de nombreuses attaques d'Aristote. Ce dernier, bien qu'il n'ait pas traité directement des arts stochastiques, offre une solution plus charitable au problème de la faillibilité. La réhabilitation des arts stochastique se fait, chez le Stagirite, par l'intermédiaire de sa réflexion sur l'éthique. Dans l'Éthique à Nicomaque, il est manifeste qu'il réplique moqueusement à Platon lorsqu'il affirme:

C'est qu'en effet, on ne doit pas chercher la même rigueur dans toutes les discussions pas plus qu'on ne l'exige dans les productions de l'art. [...], car il est d'un homme cultivé de ne chercher la rigueur pour chaque genre de choses que dans la mesure où la nature du sujet l'admet: il est évidemment à peu près aussi déraisonnable d'accepter d'un mathématicien des raisonnements probables que d'exiger d'un rhéteur des démonstrations proprement dites ${ }^{19}$.

Selon Aristote, il est illogique d'exiger des rhéteurs ou des médecins qu'ils procèdent avec une exactitude mathématique, la nature même de leur art rendant impossible de procéder de la sorte. Plutôt que de renoncer à accorder à la rhétorique et à la médecine le statut d'art, Aristote introduit un critère semblable à celui d'Hippocrate dans De l'ancienne médecine. Certains arts, dont la nature empêche une exactitude que seule la mesure ( $\mu \varepsilon \dot{\tau} \tau \rho \circ)$ peut garantir, procèdent de manière approximative, en visant le juste milieu ( $\mu \varepsilon \dot{\sigma} \sigma o v)$. C'est notamment le cas des vertus, que l'on atteint en cherchant à se préserver des écarts de tempérament extrêmes, c'est-à-dire le manque ou l'excès. Cette action qui consiste à viser le juste milieu est nécessairement approximative:

Par exemple, si ro est beaucoup, et 2 peu, 6 est le moyen pris dans la chose, car il dépasse et est dépassé par une quantité égale; et, c'est là un moyen établi d'après la proportion arithmétique. Au contraire, le moyen par rapport à nous ne doit pas être pris de cette façon: si, pour la nourriture de tel individu déterminé, un poids de ro mines est beaucoup et un poids de 2 mines peu, il ne s'ensuit pas que le maître de gymnase prescrira un poids

17. Douglas S. Hutchinson, I988, p. 37.

18. Salvatore Di Piazza, 2007, p. I 54.

19. Aristote, Éthique à Nicomaque (I094bi2-I4 \& 23-27), traduction de J. Tricot, 2007, p. 38-39. 
de 6 mines, car cette quantité est peut-être aussi beaucoup pour la personne qui l'absorbera, ou peu ${ }^{20}$.

Pour Aristote, le fait que certains arts doivent composer avec des objets qui admettent des variations n'est pas une marque de faiblesse. La nature des objets avec lesquels certains artisans doivent traiter, tels que le corps pour le médecin, ou l'âme pour le rhéteur, est changeante. En refusant de s'adapter à ces fluctuations, l'artisan risquerait d'aller à l'encontre de la raison d'être de son art, par exemple en prescrivant une trop forte dose d'un remède qui s'avèrerait dommageable pour un malade.

Il est indispensable de souligner le rapprochement entre la position aristotélicienne concernant les arts approximatifs et la manière dont la médecine est décrite dans le Corpus hippocratique, et plus particulièrement dans De l'ancienne médecine. Comme le souligne Boudon-Millot, les deux auteurs insistent grandement sur l'importance de demeurer le plus précis possible tout en tenant compte des particularités de chaque individu ${ }^{21}$. Évidemment, admettre que certains arts puissent être, par nature, approximatifs, soulève un défi supplémentaire tant pour Aristote que pour l'auteur du traité hippocratique: comment évaluer la compétence de l'artisan lorsque la nature de son art lui interdit d'être toujours constant dans ses résultats? Autrement dit, on doit pouvoir être en mesure de rendre compte de la faillibilité des arts stochastiques tout en montrant qu'il ne s'agit pas pour autant d'une défaillance de la part de l'artisan.

L'auteur du traité hippocratique répond à ce défi en distinguant la connaissance médicale générale de la connaissance des cas particuliers. La singularité de chaque cas complique fortement l'application des connaissances théoriques. Il arrive aussi qu'un patient désobéisse aux ordonnances du médecin, qu'il soit pris en charge trop tard ou qu'il soit atteint d'une maladie incurable 22 . Autrement dit, il arrive que certaines infortunes sur lesquelles le médecin n'a aucun contrôle viennent entraver la bonne conduite

20. Aristote, Éthique à Nicomaque ( I Io6a33-b6), traduction de J. Tricot, 2007, p. I IoII I. Des remarques similaires sont faites par Platon dans le premier livre de la République. L'art médical, dans la mesure où son rôle est de restituer aux malades leur santé, doit chercher ce qui est dans l'intérêt du plus faible. Pour cette raison, le médecin ne prescrit pas au malade le régime de quelqu'un de bien portant (République I 34I-342).

21. Véronique Boudon-Millot, 2005 , p. 97.

22. Joel E. Mann, 2008, p. I I I. La capacité de reconnaître les maladies incurables faisait d'ailleurs partie des compétences du médecin. Cette constance traverse une bonne partie de l'époque antique jusqu'à l'époque romaine. On peut lire dans les Aphorismes du Corpus hippocratique: «Ce que les médicaments ne guérissent pas, le fer le guérit; ce que le fer ne guérit pas, le feu le guérit; ce que le feu ne guérit pas doit être regardé comme incurable» (Aphorisme 7.87., traduction Littré IV, p. 609). Plus tard, on lira chez Aulus Cornelius Celsus ( ${ }^{\text {er }}$ siècle de notre ère): "Quand il s'agit de blessures, un médecin doit avant tout savoir reconnaître celles qui sont incurables, celles qu'il est difficile de soigner, celles dont le traitement est relativement facile. C'est en effet le premier devoir d'un homme sage que de ne pas toucher au malade qui ne peut être sauvé, et de ne pas se laisser prendre à des apparences qui l'accuseraient d'avoir tué 
d'un traitement. Pour ces raisons, l'auteur de De l'ancienne médecine prise le praticien qui «ne commettrait que de petites erreurs, la précision parfaite étant un spectacle très $\operatorname{rare}^{23}$ ». Pourtant, comme le mentionne Schiefsky dans ses commentaires sur le traité, même si les erreurs du médecin sont justifiables et excusables, il n'en demeure pas moins qu'elles restent des erreurs dues à une connaissance imparfaite. Bien que cela semble humainement inenvisageable, on peut supposer qu'une connaissance parfaite de la constitution des patients permettrait d'atteindre une précision absolue dans les traitements ${ }^{24}$.

Il s'agit d'ailleurs d'un point de rupture entre la médecine telle que dépeinte dans le Corpus hippocratique et la manière dont Aristote la présente. Pour ce dernier, l'aspect stochastique de certains arts n'est pas causé par la limitation imposée par l'objet aux connaissances de l'artisan. Autrement dit, il semble qu'un médecin pourrait parvenir à une connaissance complète de son art, appliquer le traitement qui conviendrait le mieux et tout de même échouer dans son entreprise. À première vue, cela semble tout bonnement invalider la prétention des arts stochastiques à constituer un réel savoir. Cependant, Aristote apporte une réponse intéressante au problème de la faillibilité des arts stochastiques en usant d'un argument concernant les régularités dans la nature. Dans la Métaphysique, il écrit:

Toute science se propose, en effet, ou ce qui est toujours, ou ce qui est le plus

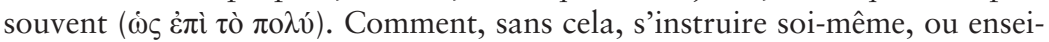
gner autrui? Il faut que la chose soit déterminée comme arrivant toujours, ou le plus souvent. Ainsi, on peut bien dire que l'hydromel est bon pour les fiévreux, le plus souvent; mais on ne pourra pas rendre compte des cas exceptionnels, dire à quel moment cet effet de l'hydromel ne se produit pas, à la nouvelle lune, par exemple: car, même ce qui arrive à la nouvelle lune arrive soit toujours, soit le plus souvent, alors que l'accident est en dehors du toujours et du plus souvent. Nous avons donc établi ce qu'est l'accident, quelle est la cause qui le produit, et aussi qu'il n'y a pas de science de l'accident ${ }^{25}$.

Ici, la médecine est incluse parmi les sciences de ce qui est le plus sou-

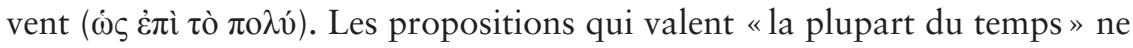
peuvent pas être universalisées, contrairement à celles qui sont «toujours" justes. En revanche, cela ne signifie pas que les propositions qui sont vraies dans la plupart des cas ne peuvent pas recevoir d'explication causale. Il y a une explication pour laquelle l'hydromel est généralement bénéfique au fiévreux. Or il arrive qu'un remède qui fonctionne habituellement échoue, auquel cas il est évident qu'il y a nécessairement une raison qui peut expli-

un homme victime de son propre destin» (Cels. 5, 26, I C-D), traduction de D. Gourevitch, I984, p. 206-207.

23. Hippocrate, De l'ancienne médecine ( $\left(\int 9\right)$, traduction de J. Jouanna, I990, p.I28.

24. Mark. J. Schiefsky, 2005 , p. I90.

25. Aristote, Métaphysique (I027a20-27), traduction de J. Tricot, 2003, p. 340-34 I. 
quer cet échec. Toutefois, bien qu'il soit possible de rendre compte de chaque échec de manière individuelle, il est impossible d'en élaborer une science, précisément parce que cela supposerait de posséder une connaissance de l'exception. C'est-à-dire être capable de prédire dans tous les cas lorsque la nature ne se comportera pas comme elle le devrait ${ }^{26}$, ce qui paraît être une impossibilité, voire une contradiction dans les termes.

Nous sommes désormais à même de répondre à la question posée un peu plus tôt concernant la possibilité d'évaluer les compétences de l'artisan lorsque la nature approximative de son art lui interdit d'obtenir invariablement du succès dans chacune de ses démarches. Pour l'auteur du traité hippocratique, le meilleur médecin est celui qui, bien qu'il procède de manière approximative, atteint le mieux sa cible. Plus encore, l'auteur insiste sur le fait que la plupart des médecins, bien qu'ils soient incompétents, composent avec des maladies bénignes où même les erreurs graves passent souvent inaperçues. C'est uniquement lorsqu'ils doivent traiter avec des maladies sérieuses où la moindre bévue entraîne des conséquences irréparables que leur incompétence devient flagrante.

Pour illustrer son propos, l'auteur de De l'ancienne médecine établit un parallèle entre le mauvais médecin et le mauvais pilote. Les erreurs de ce dernier, pour autant qu'il navigue par temps calme, sont invisibles pour le profane. Par contre, s'il doit affronter un orage, une houle violente ou des vents contraires, son inexpérience sera manifeste aux yeux de tous ${ }^{27}$. La différence entre le bon et le mauvais praticien n'est donc jaugée qu'à l'aune des cas les plus difficiles. C'est pour cette raison que l'auteur estime aussi chèrement les rares médecins qui, malgré la nature approximative de leur art, commettent peu d'erreurs, particulièrement lorsque la moindre errance peut provoquer la mort du patient.

Évidemment, Aristote croit aussi que le praticien qui commet le moins d'erreurs est plus compétent que celui qui accumule les bévues. Toutefois, il y a une limite au-delà de laquelle le médecin, même le meilleur, n'a aucune emprise; c'est le domaine de l'accidentel, pour lequel il est impossible d'avoir une science. Le bon médecin est donc celui qui, après avoir identifié l'affection, sélectionne le traitement réussissant le plus souvent dans de tels cas. Dans l'éventualité où le traitement échoue, la connaissance du praticien ne doit pas pour autant être remise en question, comme il l'explique dans les Topiques:

Nous serons en parfaite possession de la méthode quand nous en serons au même point que pour la rhétorique, la médecine et les autres techniques de même type; on ne peut pas dire en effet que de toute manière l'orateur va persuader son public, ou le médecin guérir son patient; mais à condition qu'ils

26. Robert Jim Hankinson, 2004, p. Iо.

27. Hippocrate, De l'ancienne médecine (\$9), traduction de J. Jounna, I990, p. I28- 
ne négligent aucun des moyens à leur disposition, nous pourrons dire qu'ils possèdent adéquatement leur science ${ }^{28}$.

On constate ici un nouveau point de rupture entre le traité De l'ancienne médecine et Aristote. Pour ce dernier, même si le médecin parvenait à une connaissance parfaite de son art, certains traitements échoueraient sans

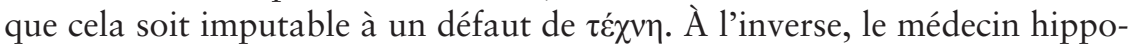
cratique, s'il venait à parfaire complètement sa connaissance de la constitution du corps et des traitements, pourrait obtenir un succès assuré. Toutefois, Hippocrate reconnaît que la nature de l'objet avec lequel le médecin doit composer l'empêche de parvenir à un tel degré de connaissance, bien qu'il doive toujours chercher à perfectionner sa technique.

Nous verrons à présent que les débats concernant la nature des arts stochastiques, leur degré de précision ainsi que leur taux de succès sont loin d'être réglés malgré ces quelques prises de position tranchées de la part d'auteurs influents. Ces questions ont continué d'occuper une place prépondérante dans les réflexions sur l'art médical dans le monde romain, entre autres chez Galien et Alexandre d'Aphrodise, dont nous allons à présent analyser les positions.

\section{Les arts conjecturaux chez Alexandre d'Aphrodise et Galien}

Malgré près de six siècles séparant les auteurs vus à l'instant de Galien et d'Alexandre d'Aphrodise, les critères permettant de distinguer les arts non stochastiques des arts stochastiques restent sensiblement les mêmes. C'est toujours le caractère faillible et le manque de précision qui caractérisent les arts stochastiques et qui contribuent par le fait même à leur statut précaire parmi les $\tau \dot{\varepsilon} \chi v \alpha 1$.

Toutefois, comme nous allons le voir à présent, ce sont les moyens de défendre les arts stochastiques qui se sont considérablement enrichis, particulièrement au contact de la philosophie stoïcienne. L’origine de la réflexion sur la faillibilité des arts chez les stoïciens se fait par l'intermédiaire de leur interrogation sur la finalité de la vie humaine. La sagesse est un art, et plus

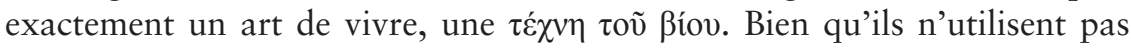
explicitement la distinction entre art stochastique et art non stochastique, il est clair que Zénon, Cléanthe et Chrysippe rangent la pratique de la sagesse du côté des arts stochastiques. Pour cause, même si le sage stoïcien vise invariablement à agir de manière vertueuse, il arrive qu'il échoue en raison de facteurs qui sont hors de son contrôle. La première solution, peu attirante, consisterait à admettre que, puisque personne n'est en mesure d'agir en tout temps de manière vertueuse, l'idéal du sage est tout simplement inatteignable. Quant à la seconde solution, elle ne semble pas plus prometteuse: en

28. Aristote, Topiques (Iorb5-II), traduction de J. Brunschwig et M. Hecquet, 20 I 5, p. 70 . 
admettant que le bonheur découle de la vie vertueuse, ce que les stoïciens reconnaissent généralement, il semble que le sage, puisqu'il échoue à agir vertueusement en tout temps, sera en proie au malheur. La réponse plutôt évidente à ce paradoxe est d'introduire une distinction entre la finalité

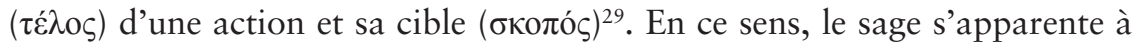
l'archer: tous deux cherchent à accomplir la finalité qui est propre à leur art. La finalité du sage est de vivre vertueusement, alors que celle de l'archer est de faire mouche. Par contre, s'il arrive que l'un ou l'autre rate sa cible, il n'en demeure pas moins que la finalité restera toujours la même. Pour cette raison, même lorsque la cible est manquée, le sage n'est pas pour autant moins sage ni moins heureux ${ }^{30}$.

\section{Alexandre d'Aphrodise}

Alexandre d'Aphrodise intègre l'analyse stoïcienne dans sa réflexion sur les arts stochastiques tout en préservant un héritage aristotélicien. Il est manifeste que, bien qu'elle soit intéressante pour le cas du sage, la position stoïcienne soulève des problèmes dans le cas des artisans. Même si toutes les actions du médecin ont pour finalité de restituer la santé d'un patient, il est nécessaire de conduire avec succès la thérapie. Sans quoi, on tombe dans la position plutôt contre-intuitive où la finalité de l'art médical n'est plus de restituer la santé du patient, mais uniquement de produire des tentatives, sans égard pour les résultats. Cette position plutôt absurde supposerait que les échecs répétés de l'art ne sont pas une raison suffisante pour contester la prétention de la médecine à être une $\tau \dot{\varepsilon} \chi v \eta$ véritable. La posture d'Aristote concernant les arts stochastiques, bien qu'elle semble mieux adaptée, pose aussi un problème pour Alexandre d'Aphrodise. Nous l'avons vu, pour Aristote, la finalité de l'art médical est de restituer la santé du patient. Lorsque le médecin échoue dans son entreprise, cela n'est pas nécessairement imputable à son incompétence, mais plutôt à la nature irrégulière du sujet avec lequel il compose. À l'inverse, il n'est pas improbable que la finalité de l'art médical soit rencontrée de manière accidentelle. Autrement dit, un médecin inexpérimenté pourrait soigner un patient simplement par chance et ainsi répondre aux exigences de l'art médical sans pour autant y être instruit. La position aristotélicienne constitue donc un problème à propos des réussites de l'art puisqu'elle échoue à fournir un critère permettant de distinguer le médecin compétent du médiocre ${ }^{31}$.

29. Inwood, I986, p. 55 I. Bien que la distinction entre $\tau \hat{\imath} \lambda$ o mulée uniquement chez Antipatros de Tarse, elle est implicitement soutenue par d'autres philosophes stö̈ciens, notamment Chrysippe. La distinction moderne consiste à souligner la différence entre l'intentionnalité des conséquences d'une action.

30. Évidemment, cela ne veut pas dire que toutes les actions du sage sont dirigées vers une seule cible: accroître son bonheur. Ce dernier n'est qu'une conséquence des actions vertueuses sans pour autant en être la motivation.

31. Ierodiakonou, I995, p. 479. 
Alexandre d'Aphrodise offre une solution ingénieuse à ces deux problèmes en optant pour une voie mitoyenne. Il distingue d'une part la fonction d'un art et, d'autre part, sa finalité. Dans le cas des arts non stochastiques, ces deux variables coïncident. Lorsque les actions employées afin d'atteindre la finalité d'un art sont accomplies en conformité avec les principes de cet art, la finalité est nécessairement rencontrée. Les cas où la finalité de l'art n'est pas rencontrée sont la conséquence d'une erreur de la part de l'artisan. Les arts stochastiques, on l'aura deviné, se comportent différemment puisque leur fonction et leur finalité ne coïncident que partiellement ${ }^{32}$. Ainsi, même lorsque l'artisan s'acquitte des tâches qu'exige son art, il ne s'ensuit pas nécessairement que la finalité sera rencontrée ${ }^{33}$.

La solution qu'apporte Alexandre aux problèmes rencontrés par Aristote et les philosophes stoïciens offre deux avantages bien nets. D'abord, il évite la position contre-intuitive des stoïciens où la finalité de l'art médical n'est plus de restituer la santé du patient, mais de tenter par tous les moyens possibles de le faire. Puis il résout du même coup le problème posé par la théorie aristotélicienne qui ne fournissait qu'un critère approximatif pour départager l'artisan compétent de l'artisan chanceux. Il faut noter que ce problème ne concerne que les arts stochastiques. Dans les arts non stochastiques, la fonction et la finalité coïncident. Conséquemment, l'accomplissement de la fonction garantit le succès d'un art. L'expert obtiendra donc du succès là où le profane échouera. Pour que ce dernier mène à bien son entreprise, il faudrait qu'il accomplisse de manière fortuite la fonction de l'art à la manière d'un expert, ce qui suppose un tour de force qu'il serait difficile de reproduire. Selon Alexandre d'Aphrodise, le médecin compétent est évidemment celui qui accomplit la fonction de son art en posant les gestes adéquats. Ainsi, il devrait atteindre la finalité de son art la plupart du temps. Quant à l'apprenti ou le profane, ils atteindront parfois la finalité de l'art de manière accidentelle. Auquel cas on saura qu'ils ne sont pas qualifiés puisque, même s'ils atteignent la finalité de l'art, ils ne l'atteignent pas en agissant en connaissance de cause, mais uniquement de manière fortuite.

Assurément, la manière dont Alexandre d'Aphrodise rend compte de la faillibilité des arts stochastiques est étroitement liée à sa théorie des futurs contingents, qu'il emprunte en bonne partie à Aristote. Par chance, un com-

32. Évidemment, il est nécessaire que la fonction et la finalité entretiennent un rapport qui n'est pas simplement accidentel puisque, comme nous l'avons vu chez Aristote, il ne peut y avoir de science des accidents. Nous verrons très prochainement comment Alexandre d'Aphrodise intègre la réflexion aristotélicienne des futurs contingents afin d'expliquer pourquoi la nature se comporte la plupart du temps d'une manière déterminée, bien qu'elle admette certaines exceptions.

33. Cette discussion sur les arts stochastiques peut être trouvée à la Quaestio 2.I6 des Quaestiones. À défaut d'une traduction française, voir la traduction anglaise de R. W. Sharples chez Bloomsbury, 2014. 
mentaire d'Alexandre d'Aphrodise sur les Premiers Analytiques vient clarifier l'explication plutôt lacunaire d'Aristote:

For some natural events are indeed proved on such a basis, and there are some arts concerned with what is in this way contingent - for instance, the conjectural arts. A doctor assumes that someone who is ill in such-and-such a way is for the most part unwell from surfeit, and that someone who is unwell from surfeit is for the most part cured by venesection; and he deduces that it is contingent that someone who is ill in this way will be cured by venesection. Taking this to be so, he performs a venesection ${ }^{34}$.

Le fait est que, pour Alexandre d'Aphrodise, les arts stochastiques utilisent des syllogismes dont les prémisses ne contiennent pas des propositions nécessaires, mais des propositions contingentes. Conséquemment, leurs conclusions ne peuvent être elles aussi que contingentes. Contrairement aux théorèmes géométriques, les théorèmes de la médecine ne sont valides que "la plupart du temps ${ }^{35}$ ».

Pour conclure, le médecin compétent est celui qui, en identifiant le mal qui affecte le patient, choisit le remède qui a le plus de chance de réussir. Dans les cas où le traitement réussit, le médecin a rempli et la fonction et la finalité de son art. Quant aux cas où la finalité n'est pas rencontrée, c'est-àdire lorsque le malade n'est pas guéri, le médecin, pour autant qu'il ait agi conformément aux principes de son art, n'est pas fautif puisqu'il ne s'agit pas d'une erreur qu'il aurait pu empêcher. Nous verrons à présent que la réponse qu'apporte Galien au problème de la faillibilité des arts stochastiques se distingue tant des solutions vues précédemment que de celle proposée par d'Alexandre d'Aphrodise.

\section{Galien et les deux «profils» médicaux}

L'importance pratiquement démesurée que Galien accorde au modèle des démonstrations mathématiques n'a, en soi, rien de déconcertant. C'est un lieu commun des savants de l'Antiquité qui se poursuit très tard dans la tradition philosophique occidentale ${ }^{36}$. Ainsi, Galien croit qu'il serait possible de mettre fin aux interminables querelles opposant les différentes sectes médicales de son époque si tous s'entendaient pour exiger des médecins le même type de démonstration qu'on exige des arithméticiens et des géomètres. Voici ce que Galien propose: "pour tout ce qui se démontre aussi dans le domaine médical, il faut tout ramener à des prémisses premières

34. Alexander of Aphrodisias, I99I, p. 98, traduction de Barnes-Bobzien-FlanneryIerodiakonou (ce commentaire porte plus exactement sur l'utilisation des syllogismes dans le cas des arts conjecturaux), pour une excellente discussion sur cette question, consulter l'article de Luca Gili (à venir).

35. Chiaradonna, 2008, p. I4.

36. Sur l'influence des mathématiques sur les disciplines scientifiques et les arts, consulter le chapitre IV de l'ouvrage de Geoffrey E. R. Lloyd: Greek Science after Aristotle (I973). 
indémontrables et qui tirent leur preuve d'elles-mêmes ${ }^{37}$ ». En usant d'une telle méthode apodictique, Galien croit qu'il serait possible d'assoir la connaissance médicale sur des conclusions irréfutables, à partir desquelles elle ne saurait que progresser ${ }^{38}$. De prime abord, cette proposition a évidemment de quoi surprendre. En effet, la prétention de réduire l'art médical à un ensemble d'axiomes peut sembler aberrante lorsqu'on considère qu'il s'agit d'une discipline où l'aspect empirique est indubitablement prépondérant. Nous montrerons à présent que la proposition de Galien, bien qu'audacieuse, n'a pourtant rien d'incongru.

Lorsque Galien suggère que la médecine, comme les mathématiques, peut procéder de manière axiomatique, il ne prétend pas qu'il soit possible de réduire l'ensemble de l'art médical à un catalogue d'axiomes. Pour Galien, les axiomes sont les premiers éléments nécessaires d'une chaîne

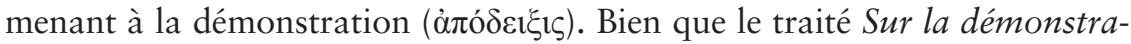

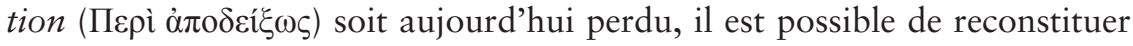
approximativement les propos de Galien par l'intermédiaire de ses autres œuvres, notamment le De methodo Medendi et le De Placitis Hippocratis et Platonis. Le médecin de Pergame distingue trois types d'axiomes. Les premiers types d'axiomes sont des principes logiques. Les seconds, qui concernent les sciences particulières, consistent à énoncer la nature de l'objet ou son essence ${ }^{39}$. Ces deux dernières catégories d'axiome ne sont pas, en elles-mêmes, suffisantes pour générer des théorèmes utiles à la médecine. Pour cause, leur caractère tautologique les prive d'un contenu empirique essentiel à toute science fondée sur l'expérience. D'où la nécessité pour Galien de postuler l'existence de propositions axiomatiques fondées sur des observations empiriques. Cette troisième catégorie d'axiomes n'est pas clairement définie par Galien, qui semble ouvert à la possibilité qu'une quantité potentiellement infinie d'énoncés puissent être des énoncés d'observations ${ }^{40}$. Nous ne souhaitons pas discuter en longueur des problèmes soulevés par la

37. Galien, Sur la méthode thérapeutique, Kühn (X, 33, L.17 - 34 L.2) traduction de J. Boulogne, 2009, p. 67.

38. Pietrobelli, 2009, p. II3.

39. Barnes, I99I, p. 7 I.

40. Ibid., p. 72. Dans son édition anglaise (aucune traduction française n'est disponible à ce jour) du De Placitis Hippocratis et Platonis (I978), Phillip de Lacy rapporte les témoignages et fragments portant sur l'œuvre de Galien. Au fragment III, intitulé De demonstratione, on peut lire: "Beginning our inquiry with the voluntary movements which are the province of that part of the soul that is specifically designated as governing, we showed that only one argument was formulated scientifically, having its premises from the essence of the very thing being investigated. It is the following "Where the beginning of the nerves is, there is also the governing part of the soul." This, the primary premise of the argument, has been agreed to by all physicians and philosophers. Its added premise, as it were, "The beginning of the nerves is in the brain" is true; but "The beginning of the nerves is in the heart" is false. You can write this latter premise, you can even say it to persons unfamiliar with anatomy; but you cannot prove it» (p. 67). On peut supposer que c'est le genre d'énoncé empirique auquel Galien référait. 
position galénique sur les axiomes et la manière de les découvrir. Ce travail a déjà été accompli par Barnes, qui souligne l'originalité de la proposition épistémologique de Galien, notamment en ce qui a trait à la possibilité d'entraîner nos perceptions et notre intellect à distinguer les axiomes véritables de ceux qui en ont uniquement l'apparence. Mais Barnes pointe aussi certaines inconsistances, entre autres: quelle nécessité y a-t-il d'aiguiser nos facultés si l'apodicticité des axiomes doit être reconnue à la première impres$\operatorname{sion}^{41}$ ? Quoi qu'il en soit, on retiendra que l'aspect stochastique de la médecine ne réside pas dans la partie apodictique de l'art médical.

Si Galien croit que l'art médical peut être axiomatisé, il ne prétend pas que l'ensemble du savoir médical peut l'être. En fait, comme le souligne Vegetti, il y a une tension constante dans l'œuvre de Galien entre ce qui apparaît comme deux profils divergents de la médecine. D’une part, une médecine haute, fonctionnant de manière axiomatique et dont l'anatomie constitue le point culminant. D'autre part, la médecine basse ou clinicothérapeutique, qui est enracinée dans une tradition plus empirique, faisant peu de cas des démonstrations bien menées ou du statut épistémologique de l'art ${ }^{42}$. Ces deux conceptions de la médecine correspondent d'ailleurs assez bien à l'opposition classique entre $\dot{\varepsilon} \pi \iota \tau \eta \dot{\mu} \mu$ et $\tau \dot{\varepsilon} \chi v \eta$. Toutefois, et contrairement à Vegetti, nous préférons opter pour l'hypothèse selon laquelle les deux manières qu'a Galien d'envisager la médecine sont conciliables ${ }^{43}$. En utilisant deux ouvrages au paradigme complètement opposé, Boudon-Millot a montré que l'embarrassante dichotomie présente dans les œuvres de Galien ne représente pas une incohérence. Ainsi, il n'y a rien de trop surprenant à voir Galien placer la médecine tantôt du côté des arts nobles, comme dans le Protreptique, tantôt la comparant à l'art du ravaudeur comme dans le $\grave{A}$ Patrophile. L'exigence scientifique cède donc parfois le pas à l'efficacité rhétorique lorsqu'il s'agit d'encenser l'art médical dans les écrits destinés aux novices ${ }^{44}$. Toutefois, cette explication, bien qu'intéressante, demeure plutôt insatisfaisante. L'idée que la justesse scientifique soit parfois minorée à des fins rhétoriques explique difficilement nos propos tenus plus haut concernant la possibilité d'obtenir des vérités premières en médecine.

41 Ibid., p. 78. Plusieurs hypothèses ont été avancées afin de régler ce paradoxe apparent chez Galien. Barnes en énonce lui-même quelques-unes, notamment la perte du traité galénique De la démonstration dans lequel ces problèmes étaient très probablement traités. Dans son article de 1988, Galen on the Foundations of Science, Robert J. Hankinson apporte aussi des pistes de solution intéressantes.

42. Vegetti, I98I, p. 58.

43. L'hypothèse voulant qu'une certaine conciliation soit possible est aujourd'hui plus répandue. À ce sujet, consulter l'article de S. Fortuna, «La definizione della medicina in Galeno» (I987) ou celui de P. N. Singer «Levels of Explanation in Galen» (I997). Pour notre part, nous privilégierons l'utilisation d'une source en français, soit celle de Véronique Boudon Millot, «Art, science et conjecture chez Galien» (2003).

44. V. Boudon-Millot, 2003 , p. 276. 
La solution plutôt inusitée de Galien pour capturer la nature d'un art qui semble échapper à toute classification sera de postuler que l'art médical n'est ni une $\dot{\varepsilon} \pi 1 \sigma \tau \eta \dot{\mu \eta}$ ni une $\tau \dot{\varepsilon} \chi v \eta$, mais une participation des deux: «ce qui fonde le lien art-savoir c'est précisément l'existence d'une méthode ${ }^{45}{ }$. Au premier abord, cet argument a évidemment l'apparence d'une solution ad hoc. Nous verrons cependant que la manière qu'a Galien de justifier sa proposition est tout à fait légitime:

[...] sachant bien qu'il n'est possible d'acquérir la science d'aucun art sans imposer de méthode par l'intermédiaire de ce qu'on appelle généralement des "théorèmes", et un entraînement par l'intermédiaire du détail des exemples. En effet, ni il n'est possible de faire ce qu'il faut sans s'être livré à de multiples espèces d'exercices en entrant dans les détails sur les personnes souffrantes, ni la possibilité n'est donnée, sans la connaissance générale, que l'exercice luimême se fasse convenablement. Car la méthode réside dans ces observations générales et l'entrainement dans le détail des cas particuliers ${ }^{46}$.

Ce passage nous semble être un excellent exemple de l'inclination de Galien envers l'école dogmatique. Parmi les trois sectes médicales présentes à son époque, Galien refuse d'accepter les «idioties » des méthodistes ${ }^{47}$, qui prétendent que la médecine est une science pouvant être apprise en six mois. Puis, il condamne aussi la secte empirique en raison de son rejet des outils logiques dont Galien fait lui-même usage. Pour les empiriques, la médecine est un art dont l'apprentissage et la pratique sont essentiellement une question d'expérience ${ }^{48}$.

Aussi Galien ne semble-t-il pas considérer qu'il est problématique de

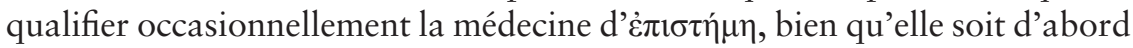
et avant tout une $\tau \varepsilon^{\prime} \chi v \eta$. En circonscrivant le domaine de l'art médical aux seuls cas relevant de sa compétence, il évite la critique aristotélicienne selon laquelle il ne saurait y avoir de science des particuliers. Cette définition lui permet aussi d'ignorer provisoirement le problème de la faillibilité des arts sur lequel tous les auteurs considérés précédemment ont achoppé ${ }^{49}$. Toutefois, cette difficulté ne saurait être passée complètement sous silence. Face aux échecs avec lesquels les médecins doivent composer quotidiennement, le médecin de Pergame est contraint d'aborder le problème de la faillibilité de l'art médical. Après tout, si la médecine n'atteint pas toujours sa cible, est-il toujours légitime de la qualifier d'art ou pire, de science? Face à cette

45. Ibid., p. 28 I.

46. Galien, Sur la méthode thérapeutique, Kühn (X, 628, L.10 - L.17) traduction de J. Boulogne, 2009, p. 517.

47. Voir plus bas l'extrait du traité Sur la méthode thérapentique, p. I4.

48. À propos des débats épistémologiques entre les sectes médicales, consulter les chapitres XIV, XV et XVI de l'ouvrage Essays in Ancient Philosophy (1987) de Micheal Frede. 49. V. Boudon-Millot, 2003, p. 288. 


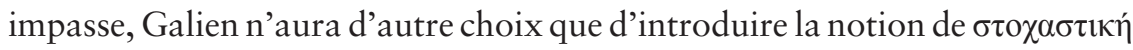
qui servira de relai entre l'غ̇ $\pi \iota \sigma \tau \mid \mu \eta$ et la $\tau \dot{\varepsilon} \chi v \eta^{50}$.

\section{Diagnostic et conjecture technique}

C’est la nécessité de recourir à la conjecture, plutôt que sa faillibilité, qui fait de l'art médical un art stochastique selon Galien ${ }^{51}$. Cette contrainte est particulièrement accrue pour ceux qui pratiquent la thérapeutique: "La thérapie est la conséquence aussi bien du diagnostic que du pronostic. Il faut en effet diagnostiquer le présent et, à partir de là, pronostiquer le futur, si l'on veut recourir à un remède efficace ${ }^{52}$." Certaines affections permettent un diagnostic clair à partir duquel on pourra prescrire une thérapie conséquente. Comme Galien l'écrit dans son traité sur l'hygiène, il est toutefois beaucoup plus fréquent de voir des affections où le diagnostic nécessite de faire usage de la «conjecture technique »: "Most diseases have, however, a conjectural diagnosis, so as not to be known from a collection of defined symptoms $s^{53}$.» Pour diagnostiquer une affection, le médecin doit s'employer à découvrir les diathèses ${ }^{54}$ responsables du mauvais fonctionnement des parties du corps. Cela requiert évidemment de posséder une méthode:

Je voyais que la secte empirique s'attachait à se rappeler et à imiter non pas les cas rares, mais les cas fréquents, et que par suite elle négligeait non pas seulement le traitement des diathèses rares, mais encore les signes indicateurs de ces diathèses. En conséquence, j'ai cherché d'abord comment il faut les diagnostiquer, et j'en ai découvert quelques-unes qui présentent un diagnostic scientifique, d'autres qui étaient subordonnées à la conjecture dite technique, et dont, en conséquence, le diagnostic se rectifiait souvent; car telle est la puissance de la conjecture technique. Mais pour ces diathèses, et avant cela, pour celles des diathèses rares qui présentent un diagnostic scientifique, je trouvai qu'elles exigent toujours le diagnostic des parties affectées ${ }^{55}$.

Au passage, Galien en profite pour réitérer son différend avec la secte empirique. Nous reviendrons plus bas sur ce qu'il entend lorsqu'il affirme qu'il est indispensable d'identifier les parties affectées afin d'établir un dia-

50. Ibid., p. 289

51. Bien que Galien refuse d'aborder le problème de la faillibilité de l'art médical de manière directe, il n'en demeure pas moins que la nécessité de recourir à la conjecture représente une tentative d'expliquer le statut faillible de la médecine. C'est cette nécessité qui fait de la médecine un art stochastique plutôt que l'inconsistance de ses résultats. En ce sens, il est juste de dire que Galien procède à rebours des autres auteurs.

52. Galien, Sur la méthode thérapeutique, Kühn (X, 693, L.14 - L.17) traduction de J. Boulogne, 2009, p. 567.

53. Galen, Hygiene, Kühn (VI, 355, L.8 - L.9), translated by I. Johnston, 2018, p. 91.

54. Hankinson en donne la définition suivante: "A disposition (diathesis) is a nonpermanent, but equally non-ephemeral, temperament of the body; and these dispositions are responsible for the well- or ill-functioning of its various systems" Hankinson, 2008, p. 23 I.

55. Galien, Des lieux affectés, Kühn (VIII, 145, L.11 - L.17) traduction de Daremberg, I 856 , p. 547 . 
gnostic. Avant cela, nous désirons mettre au clair la position que défend Galien en ce qui a trait au choix de la thérapie appropriée, tout en la contrastant avec les propos d'Alexandre d'Aphrodise.

Pour ce dernier, nous l'avons vu, le médecin remplit son office dès lors que, pour une affection particulière, il applique la thérapie réussissant le plus fréquemment. On notera que cette position épistémologique s'apparente à bien des égards à celle de la secte empirique dont Galien se dissocie. Pour cause, il souscrit à une hypothèse épistémologique plus contraignante qui présuppose que le médecin doit s'efforcer de connaître l'idiosyncrasie du malade afin d'améliorer le choix de la thérapie:

Il faut examiner la nature de la personne souffrante et pour chaque individu il existe une thérapie particulière; et en outre, troisième constat patent, puisque ce qui est particulier à la nature humaine est inexprimable et inintelligible même pour qui a la science la plus rigoureuse, celui-là serait le meilleur médecin de tous les états maladifs pris individuellement qui se donnerait une méthode grâce à laquelle il diagnostiquerait leur nature et aurait pour visée les médicaments propres à la particularité de chaque nature. Mais s'imaginer qu'il existe un traitement commun à tout le monde est la dernière des sottises; or c'est précisément l'opinion des champions de la stupidité que sont les Méthodistes. Et ils ont affirmé que c'est pour cette raison que tous les théorèmes de la médecine tiennent debout. C'est-à-dire présentent pour les enquêtes de la connaissance un caractère scientifique et sûr. La connaissance, disent-ils, est un certain art des "états corporels généraux ", et non pas des particularités, comme s'ils appliquaient leur thérapie au genre humain en général, et non pas aux hommes pris individuellement. Donc, de même que dans tout le reste, ils ont, dès le point de départ, fait un faux pas, de même est-ce également le cas ici: car ce n'est pas le genre humain en général qui reçoit une thérapie, mais chacun d'entre nous, avec bien évidemment des différences de crase et de nature. Ils s'imaginent qu'il existe pour tout le monde une thérapie unique. Pour ma part, si j'allais jusqu'à savoir exactement la nature de chacun, je serais personnellement l'égal d'Asclépios, tel que je le conçois; mais, puisque c'est impossible, $\mathrm{j}$ 'ai, en tout cas, décidé de m'exercer personnellement à m'approcher de cet idéal au maximum des capacités humaines, et c'est ce que je recommande aux autres ${ }^{56}$.

Autrement dit, l'idiosyncrasie de l'individu interfère avec toute tentative de formuler une théorie médicale qui permettrait de déduire a priori, pour chaque patient, quel traitement doit être adopté. Puisque la thérapeutique est un art qui s'occupe du particulier, il est impossible de faire abstraction des particularités des malades dans le choix du traitement. Les

56. Galien, Sur la méthode thérapeutique, Kühn (X, 205, L.16 - 207, L.5) traduction de J. Boulogne, 2009, p. 195-196. Nous ne sommes pas les premiers à relever ce passage essentiel du MM. Pour de plus amples commentaires au sujet de l'insaisissabilité des natures individuelles, on pourra consulter Ballester (1994), Ierodiakonou (1995), Boudon-Millot (2003) et Di Piazza (2016). 
différentes branches théoriques de la médecine, notamment la nosologie, peuvent tout de même réduire considérablement le nombre de traitements que le médecin aura à envisager en fonction des symptômes qu'il observe. Toutefois, comme nous l'avons déjà mentionné, le diagnostic, le pronostic ainsi que la sélection finale de la thérapie nécessitent souvent de recourir à la conjecture. Les conjectures propres à l'art médical, que Galien désigne

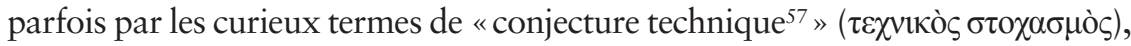
constituent le moyen auquel le médecin doit recourir lorsque les signes qu'il observe ne permettent pas d'établir un diagnostic scientifique.

De plus, le problème de la quantification fait ressurgir le caractère stochastique de l'art médical, même après le choix de la thérapie finale. Même si l'art médical permet de déterminer, suivant la nature de l'affection, quels régimes ou quels remèdes porteront le plus de secours au patient, c'est

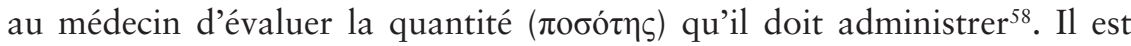
donc indéniable que la conjecture technique possède un rôle crucial dans la pratique de la médecine chez Galien. Aussi tenterons-nous maintenant d'en fournir une définition la plus exacte possible.

\section{Le diagnostic scientifique}

Paradoxalement, la première étape vers une meilleure caractérisation de la conjecture technique requiert de la contraster avec le diagnostic scientifique que nous avons mentionné plus haut. La différence réside principalement dans leur statut épistémologique. Galien définit la conjecture technique comme "un moyen terme entre la connaissance exacte et l'ignorance complète $^{59}$ ». À celle-ci, il oppose fréquemment le diagnostic scientifique, qui est précis et exact. Ce dernier se divise en deux types, comme l'a démontré Fortuna $^{60}$. Certains diagnostics scientifiques sont obtenus par déduction, cela arrive notamment pour certaines affections possédant un ensemble de symptômes distinctifs qu'elles ne partagent avec aucune autre maladie.

Toutefois, et ce point crucial pour mettre en évidence la nécessité d'un diagnostic "au cas par cas", il est plus fréquent que le médecin observe pendant son auscultation des symptômes qui sont communs à plus d'une maladie. Auquel cas il devra procéder par élimination, jusqu'à ce qu'il réussisse à isoler la cause de l'affection. Galien présente de nombreux exemples où il est confronté à des cas de la sorte. Entre autres, lorsque le médecin observe un gonflement de la vessie dû à la rétention d'urine, deux explica-

57. L'expression "conjecture technique" est très fréquente chez Galien, voir entre autres dans Kühn (I, 353, L.I3) (VI, I29, L.7 \& L.9) (VIII, I4, L.IO). En revanche, l'expression «art stochastique " n'est employée qu'une seule fois par Galien, en Kühn (XVIIB, 349, L.5).

58. S. Di Piazza, 2016, p. 785.

59. Galien, De locis affectis, Kühn (VIII, 14, L.10 - L.12) traduction de V. BoudonMillot, 2003, p. 289.

60. S. Fortuna, 200I, p. 295. 
tions sont possibles: la paralysie de la fonction excrétrice ou l'obstruction de l'uretère. Pour déterminer la cause de l'affection, le médecin place le patient debout et presse sur la vessie. Si de l'urine sort, il s'agit d'une paralysie, dans le cas contraire, on peut en conclure qu'il s'agit d'une obstruction ${ }^{61}$. Bien que le traité De locis affectis soit entièrement consacré à montrer comment établir des diagnostics précis, Galien est conscient qu'une telle démarche ne saurait être exhaustive.

Les signes précurseurs rendent cette distinction manifeste pour un homme versé dans le diagnostic. Car certaines particularités qu'on ne peut pas énoncer s'ajoutent aux symptômes susceptibles d'être énumérés, et confirment le diagnostic des parties affectées. J'exposerai seulement les bases, pour ainsi dire, du diagnostic, qui serviront aux travailleurs à apprendre par eux-mêmes ces particularités qu'on ne saurait dire ${ }^{62}$.

En somme, le diagnostic scientifique est uniquement possible lorsque le médecin a accès à des symptômes "qui manifestent clairement la propriété de la substance affectée $e^{63} »$. Dans ces cas, il sera en mesure de progresser de manière inférentielle en convertissant les signes indicatifs (

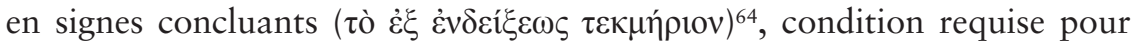
l'établissement d'un diagnostic scientifique. Cette manière de procéder alliant le raisonnement aux observations empiriques permet au praticien de connaître la nature de l'affection ainsi que sa localisation ${ }^{65}$. Le diagnostic scientifique se caractérise donc par la possibilité qu'il a d'être confirmé. En revanche, Galien prévient que l'établissement d'un diagnostic scientifique n'est pas toujours possible: "Ne cherche donc pas en chaque disposition physique des signes tels que ceux qui appartiennent à la pleuritis ou à la dysenterie. En effet, le diagnostic des états maladifs de cette sorte est scientifique $^{66}$. " Lorsque le diagnostic scientifique n'est pas possible, c'est-à-dire lorsque les symptômes ne permettent pas d'isoler une maladie particulière ou de départager parmi plusieurs affections possibles, le médecin devra obligatoirement recourir à la conjecture technique.

\section{La conjecture technique}

Le diagnostic établi à l'aide de la conjecture technique s'oppose donc aux diagnostics scientifiques en ceci qu'il n'atteindra jamais le degré de certitude

61. Galien, De locis affectis, Kühn (VIII, 7, L.8 - L.14).

62. Galien, Des lieux affectés, Kühn (VIII, 366, L.13 - L.18) traduction de Daremberg, I 856, p. 646.

63. Ibid., Kühn (VIII, 18, L.14 - L.15) traduction de Daremberg, I856, p. 477.

64. Galien, In Hippocratis prognosticum, Kühn (XVIIIB, 307, L.13 - L.14).

65. L. García-Ballester, I994, p. I665.

66. Galien, Sur la méthode thérapeutique, Kühn (X, 860, L.8 - L.10) traduction de J. Boulogne, 2009, p. 693. 
que peuvent avoir ces derniers ${ }^{67}$. Malheureusement, comme nous l'avons déjà mentionné plus haut, la plupart des maladies doivent être diagnostiquées de manière conjecturale. Pour poursuivre avec un exemple que nous avons déjà utilisé, il arrive que le médecin soit aux prises avec un patient en proie à la rétention d'urine sans toutefois que la vessie soit gonflée. Auquel cas, il est impossible de déterminer si l'affection est causée par l'obstruction des reins ou l'obstruction de l'uretère, que ce soit par des calculs ou en raison d'une inflammation ${ }^{68}$. Ce sont tout particulièrement les maladies affectant les organes internes qui requièrent d'utiliser la conjecture. Puisque les médecins de l'Antiquité ne disposaient pas des moyens nécessaires pour observer le fonctionnement des organes internes, il était souvent nécessaire de conjecturer leur bon ou leur mauvais état à partir de signes externes.

Cependant, parmi les autres organes internes aucun ne m'a jamais fourni un diagnostic clair. Il faut pourtant essayer, dans la mesure du possible, de diagnostiquer leur bon ou leur mauvais état, même si cela ne repose pas sur un savoir sûr, mais du moins sur quelque conjecture propre à notre art [...]. Et j'en ai observé un autre présentant un état tout entier sujet au phlegme et vomissant chaque jour de la bile d'un jaune pâle. J'ai alors compris qu'il fallait observer ses excréments et ils contenaient une petite quantité de bile. J'ai donc conjecturé, d'après cet indice, que le canal qui faisait dériver la bile n'en envoyait pas une faible part dans le pylore de l'estomac, comme cela se révèle être le cas chez quelques êtres vivants. Et à partir de ces exemples, l'on voit bien que dans ce qui est invisible aux sens, concourent grandement à un diagnostic, aussi bien la connaissance tirée des révélations de l'anatomie que la découverte des fonctions et de l'utilité des organes. Celui qui veut donc être capable d'établir un diagnostic pour des corps frappés de défaut dans les conditions évoquées à l'instant, doit s'exercer aux séances d'anatomie et à la découverte des fonctions et de l'utilité des organes ${ }^{69}$.

Il convient à présent de se pencher sur l'intérêt que peuvent avoir la connaissance de la fonction des organes. Dans l'exemple ci-dessus, le médecin privé de connaissances anatomiques sera dans l'impossibilité de diagnostiquer l'affection. D'abord, parce que performer un diagnostic conjectural de la sorte requiert de savoir que le rôle du cholédoque est de conduire la bile vers le duodénum. Ensuite, parce que si l'on ignore que c'est le pylore qui fait communiquer l'estomac et le duodénum, il est inenvisageable de comprendre pourquoi une petite quantité de bile peut se retrouver

67. Cela n'est pas tout à fait juste. Il est possible de confirmer (ou d'infirmer) un diagnostic conjectural, mais uniquement de manière rétrospective. Après l'établissement d'un diagnostic conjectural, le médecin prescrira une thérapie selon ce qu'il croit être la nature de l'affection. Si la condition du patient s'améliore, il en conclura que son diagnostic était exact, sinon, il devra rectifier ses conjectures.

68. Galien, De locis affectis, Kühn (VIII, 18, L.5 - L.16).

69. Galien, Ars medica, Kühn (I, 353, L.10 - L.10 \& 354, L.4 - L.16) traduction de V. Boudon-Millot, 2002, p. 332-333. 
dans les excréments lorsque le cholédoque est endommagé. Former un diagnostic de la sorte n'est donc uniquement possible que pour le médecin instruit aux séances d'anatomie puisque lui seul peut établir quelle est la partie affectée à partir des symptômes qu'il observe. Il s'agit d'ailleurs d'un point commun entre le diagnostic scientifique et le diagnostic subordonné à la conjecture technique ${ }^{70}$; tous deux requièrent de connaître la fonction des organes. Cette nécessité s'accentue particulièrement lorsque le médecin est aux prises avec un ensemble de symptômes qu'il n'a jamais observés auparavant. Dans un tel cas, Galien propose une méthode d'investigation:

Je recherche toujours quel lieu affecté primitivement ou sympathiquement a produit la lésion de la fonction, et, quand je suis certain d'avoir découvert la partie, je recherche immédiatement la diathèse de cette partie, puis de ces deux notions, je tire l'indication de tout le genre de traitement à adopter relativement à la découverte des substances convenables, à la quantité et à la qualité des remèdes, considérant en même temps l'âge et la nature du malade, la saison et le pays, et toutes les particularités qui ont été déjà souvent rapportées dans l'explication des livres d'Hippocrate. Or le lieu affecté, si nous nous rappelons ce qui a été dit dans les deux livres précédents, est reconnu par les excrétions, par les excroissances survenant sur les parties affectées et par la lésion des fonctions, ce qui comprend les formes et les couleurs contre nature ${ }^{71}$.

La conjecture technique est donc possible en vertu d'une méthode de recherche propre à l'art médical doublée d'une bonne connaissance de l'anatomie. De surcroît on peut affirmer, comme le fait Di Piazza, qu'il y a une césure nette chez Galien entre conjecture non technique et conjecture technique. Comme cela a déjà été mentionné auparavant, cette dernière est uniquement praticable pour l'individu instruit aux ressources de l'art médical ${ }^{72}$. D'ailleurs, c'est cet ancrage technique propre à certaines conjectures qui permet à Galien de défendre le statut scientifique de la médecine malgré le recours à des mesures approximatives ${ }^{73}$. Aussi la définition de la conjecture technique fournie par Galien dans le De Locis Affectis comme étant «un moyen terme entre la connaissance exacte et l'ignorance complète» ne saurait-elle être définitive. Cette définition plutôt embryonnaire semble en

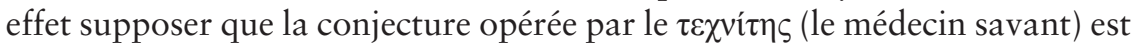
un procédé analogue au lancer d'une pièce de monnaie, la compétence n'ayant finalement rien à y voir. Auquel cas le profane n'aurait rien à envier au plus expérimenté des médecins, ce qui invaliderait instantanément le

70. Nous ne soutenons pas que les connaissances anatomiques sont aussi importantes pour les diagnostics scientifiques que pour les diagnostics établis conjecturalement. Ces derniers en profitent davantage puisqu'ils sont constitués de cas souvent atypiques pour lesquels il n'y a aucun antécédent.

71. Galien, Des lieux affectés, Kühn (VIII, 146, L.10 - 147, L.5) traduction de Daremberg, I 856 , p. 547 .

72. Galien, Hippocratis de victu acutorum commenaria, Kühn (XV, 585 -, L.7 - L.10).

73. S. Di Piazza, 20I6, p. 787. 
statut technique de l'art médical. Notre analyse du statut épistémologique de la conjecture technique selon Galien requiert donc d'être approfondie. Bien que cela ne soit jamais formulé explicitement, il est patent que le degré de plausibilité d'une conjecture va de pair avec l'expertise du médecin qui l'émet ${ }^{74}$. Cela est d'autant plus vrai lorsque le médecin doit estimer les quantités de nourriture, de breuvage, de repos ou d'exercice qui seront bénéfiques à un patient ${ }^{75}$. Si la possibilité d'émettre des conjectures techniques repose sur les connaissances anatomiques du médecin, leur amélioration est avant tout une question de pratique ( $\tau \rho \iota \beta$ ) et d'entraînement ${ }^{76}$.

En outre, l'expertise du médecin est aussi déterminante dans l'établissement des pronostics ${ }^{77}$. Ces derniers constituent une part essentielle de l'art médical puisqu'ils permettent d'estimer la durée des maladies, leur paroxysme et leur terme ${ }^{78}$. Toutefois, Galien met en garde ses contemporains contre la conception erronée voulant que l'établissement d'un pronostic soit toujours possible. Poser un pronostic nécessite que certains faits adviennent de manière assurée dans le futur ${ }^{79}$. Cependant, plusieurs facteurs

74. S. Fortuna, 200I, p. $30 \mathrm{I}$.

75. À cet effet, on peut lire dans le De sanitate tuenda, Kühn (VI, I3 I, L.3 - L.I I): For if something did escape notice at the beginning, this will be made exact when taught by expe-

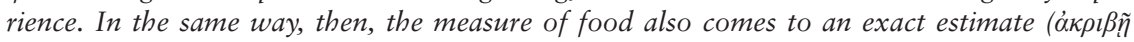
$\sigma \tau o \chi \alpha \sigma \mu \dot{v})$, although there is no way it can be known at the beginning. But the experience of each day and the recollection of the amount of food and exercise when the one in charge is not remiss in his observations, but always remembers how much food was digested after how much exercise, leads to the recognition of a more exact knowledge over time (trad. Johnston, 2018, p. I9I).

76. L. García-Ballester, I994, p. I666.

77. Jusqu'ici, nous avions volontairement omis de traiter de ce thème afin de ne pas surcharger le texte d'un aller et retour constant entre le problème du diagnostic conjectural et celui de l'établissement des pronostics.

78. L'établissement des pronostics est étroitement lié au problème de la quantification que nous avons mentionné plus haut. Dans le Ad Glauconem de Methodo Medendi Kühn (XI, 31 L.1 - 32 L.3), on peut lire: "La remarque la plus importante de toutes, la plus courte à énoncer n'est pas encore faite. Quelle est-elle? C’est le degré de la maladie et de la force du malade. La chose n'exige qu'un mot d'indication, mais elle est de la plus grande utilité. En effet, il n'est pas possible de bien pronostiquer sans calculer exactement le degré où en est chacune des choses précitées. La maladie est-elle mortelle ou ne l'est-elle pas? Quand est-il plus probable que le sujet mourra ou guérira de l'affection? C'est ce que vous ne pourrez ni prévoir ni prédire, si vous ne commencez pas par examiner attentivement le degré où se trouvent toutes les choses susdites, pour rapporter ensuite sous deux chefs la maladie même et la force du malade. En effet, si la force est assez grande pour surmonter la maladie, nécessairement l'individu sera sauvé; si le contraire existe, il mourra infailliblement. N'examinez donc pas la maladie seulement dans sa nature, mais encore dans son intensité. Cette connaissance exige une longue pratique, tant pour d'autres raisons que par cette circonstance qu.on ne peut décrire ni expliquer verbalement l.intensité de chaque affection. Si nous avons quelque talent, nous n'arrivons pas à cette exactitude d'appréciation par une cause autre que par une grande habitude à juger de la quantité » (traduction de Daremberg, I 856, p. 722-723).

79. Dans le De diebus decretoriis, Galien explique comment l'établissement d'un pronostic fiable est étroitement relié aux phases d'une maladie: "Your conjecture about the days 
pouvant influencer le cours de la maladie sont contingents, aussi banals soient-ils, tels que des aboiements de chiens qui priveraient le malade de $\operatorname{repos}^{80}$. Cela vaut aussi pour le déplacement d'une humeur superflue, duquel il peut résulter aussi bien la mort du patient qu'une guérison inattendue: "Aussi, dans de telles constitutions, ne peut-on établir de pronostic que ce qui est mal assuré et incertain, sans qu'il soit possible de découvrir avec exactitude quelle fin en résultera ${ }^{81}$. "C'est uniquement lorsque le médecin dispose d'un point fixe et certain que le pronostic devient plus fiable. Par exemple, s'il s'assure que les humeurs superflues ne s'écoulent plus, il sera en mesure, par l'intermédiaire de la conjecture technique, de déterminer le moment de leurs coctions ${ }^{82}$. La conjecture technique s'avère donc être un outil indispensable qui permet au médecin de s'approcher de la vérité lorsque les autres ressources de l'art font défaut ${ }^{83}$.

\section{Conclusion}

La revue des positions d'Hippocrate, de Platon, d'Aristote, d'Alexandre d'Aphrodise et de Galien montre que la discussion sur le caractère stochastique de la médecine n'a cessé de se développer. En effet, tandis qu'il est possible de douter qu'Hippocrate avait développé une réflexion sur la conjecture elle-même, nous avons vu que le sujet était au centre des essais de caractérisation de la médecine comme science chez les autres auteurs. L'attitude de Platon envers la médecine est mitigée. Il semble successivement la prendre comme modèle à imiter, pour ensuite la ranger du côté des arts conjecturaux en raison de son manque de précision, qui est dû aux mesures approximatives par lesquelles elle procède. Bien qu'ils ne traitent pas explicitement de la conjecture, Aristote et Alexandre d'Aphrodise affichent une attitude plutôt favorable à l'égard des arts stochastiques. Contrairement à Platon, ils reconnaissent qu'il est inconséquent de requérir de tous les arts le même degré d'exactitude. Pour certains arts, la frontière imposée par le domaine de l'accidentel interfère avec la possibilité d'obtenir invariablement du succès. L'approche du médecin de Pergame, à l'instar de celle d'Hippocrate, est beaucoup plus ancrée dans la pratique de l'art médical. Tous deux soutiennent que c'est avant tout l'aptitude permettant de juger des quantités

from the fourth day to the seventh day is weak, permitting varied interpretations, however in the days from the seventh to the eleventh day your conjecture is more reliable, and closer to not being (merely) probable in many aspects. For if you see the signs indicating that the illness has nor been concocted, and indicating that the illness is lengthening out..." (Kühn, IX, 838, L.11 - L.13, traduction de l'arabe par G. M. Cooper, 2016, p. 222).

80. V. Boudon-Millot, 2003, p. 294.

81. Galien, De constitutione artis medicae, Kühn (I, 290, L.17 - 291, L.3) traduction de V. Boudon-Millot, 2003, p. 294.

82. V. Boudon-Millot, 2003, p. 295.

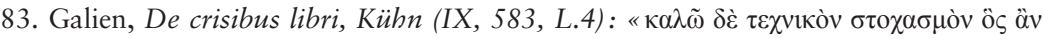

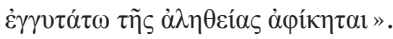


qui détermine l'excellence du médecin. Toutefois, la médecine ne s'arrête pas après Hippocrate. L'ampleur du Corpus légué à la postérité atteste de l'ardeur que Galien a déployée durant sa vie afin de faire progresser l'art médical. Cet effort se traduit notamment par la position finement élaborée sur «l'art de conjecturer ", qui outrepasse sans doute possible les réflexions des philosophes sur les arts stochastiques.

Au terme de cette étude, nous conclurons par une remarque plutôt paradoxale: peu de choses peuvent être dites à propos de la conjecture technique. Ce constat pessimiste préfigure d'ailleurs dans les premières pages du Ad Glauconem de methodo medendi:

Outre la nature commune à tous les hommes, ô Glaucon, le médecin doit connaître encore la nature individuelle de chacun. Il y a longtemps qu'Hippocrate a donné cet excellent précepte, auquel je m'efforce, comme tu le sais, de me conformer dans l'exercice de l'art. Mais il n'est pas possible d'écrire sur la nature particulière de chaque individu, comme sur la nature commune à tous; les écrits se comportent autrement que les faits, surtout dans le sujet que je me propose de traiter ${ }^{84}$.

En outre, même si Galien reconnaît que les diagnostics, les pronostics et les mesures sont, pour la plupart, déterminés à l'aune de la conjecture technique, c'est un sujet dont il traite rarement. Lorsqu'il traite de la conjecture technique, c'est généralement pour la contraster avec le diagnostic scientifique. On ne s'étonnera donc pas de constater que le De locis affectis, où Galien entreprend de montrer comment établir le diagnostic des parties affectées, est entièrement consacré aux diagnostics scientifiques. Un seul exemple de diagnostic conjectural y est évoqué pour lequel Galien nous dit qu'il est impossible de déterminer la cause de l'affection ${ }^{85}$.

Autrement dit, le diagnostic scientifique possède une nature suffisamment générale qui le rend valide pour tous les hommes, à quelques exceptions près. En contrepartie, le caractère particulier de la conjecture technique rend vaine toute tentative d'en inférer une forme générale. On peut s'imaginer en quoi consisterait cette tâche de Sisyphe, établir pour chaque individu une théorie de la conjecture technique. Aussi se retrouverait-on avec une théorie de la conjecture technique chez Socrate et une autre pour Callias, l'une et l'autre n'étant pas substituables puisqu'elles doivent tenir compte des singularités propres à la nature de chacun; Aristote avait déjà montré qu'une telle idée relevait d'une mécompréhension des arts comme la médecine, la navigation ou la rhétorique. À partir de cette constatation, il

84. Galien, Sur la méthode thérapeutique, À Glaucon, Kühn (XI, 1, L.1 - L.8) traduction de Daremberg, I 856 , p. 706.

85. Il s'agit du cas que nous avons présenté plus haut où le patient est en proie à la rétention d'urine sans que sa vessie montre des signes de gonflement. Le cas échéant, il est impossible de déterminer si l'affection est causée par une obstruction ou une inflammation, soit des reins soit de l'uretère. 
est possible de conclure avec Galien que le domaine d'application de la conjecture technique est entièrement circonscrit par celui du diagnostic scientifique. C'est toujours à ce dernier que le médecin aspire. Lorsque cela est impossible, il devra considérer toutes les particularités pertinentes qui composent le cas et tenter d'établir, par l'intermédiaire de la conjecture technique, un diagnostic aussi précis que possible. Ce faisant, il réduira autant que possible la part d'incertitude qui est introduite dans l'art médical par la complexité du corps humain et des conditions d'existence de chaque humain en particulier ${ }^{86}$.

\section{Bibliographie}

Textes anciens

Alexandre of Aphrodisias. On Aristotle's "prior Analytics 1.1-7”, Ithaca, Cornell University Press, I991, p. 252.

Aristote. Éthique à Nicomaque, Paris, Vrin, 2007, p. 577.

- Métaphysique, Paris, Vrin, 2003 , p. 877.

- Topiques, Réfutations sophistiques et Organon V-VI, Paris, Flammarion, 2015, p. 446.

Cooper, Glen, M. Galen De diebus decretoriis from Greek to Arabic..., S.I., Routledge, 20I6, p. 6I 5 .

Daremberg, Charles. CEuvres anatomiques, physiologiques et médicales de Galien..., Paris, J.-B. Baillière, I 854-I 856 , 2 vols.

Galien. On the Doctrine of Hippocrates and Plato, Berlin, Akadémie Verlag, I978, p. 83 I.

—. Exhortation à l'étude de la médecine \& Art médical, Paris, Les belles lettres, 2000 , p. 454.

-. Méthode de traitement, Paris, Gallimard, 2009, p. IоI9.

—. Le médecin. Introduction, Paris, Les belles lettres, 2009, p. 232.

-. Hygiene volume I, Cambridge, Harvard University Press, 20I 8, p. 5 I 5.

-. Hygiene, Volume II: Books 5-6. Thrasybulus. On Exercise with a Small Ball, Cambridge, Harvard University Press, 20I 8, p. 4 OI.

Hippocrate. De l'ancienne médecine, Paris, Les belles lettres, I990, p. 237.

Hippocrates. Prognostic. Regimen in Acute Diseases. The Sacred Disease. The Art. Breaths. Law. Decorum. Physician. Dentition. Cambridge, Harvard University Press, I929, p. 335.

—. On Ancient Medicine, Boston, Brill, 2005, p. 4I 5 .

Kühn, C. G. Galeni Opera Omnia, Leipzig: Teubner, I 833, 20 vols.

Littré, Emile. ed. CEuvres complètes. JB Baillière, 1861, 10 vols.

Platon. CEuvres complètes, Paris, Flammarion, 20I I, p. 2234.

\section{Textes contemporains}

Allen, James. «Failure and Expertise in the Ancient Conception of an Art» in T. Howoritz and A. Janis, eds., Scientific Failure, Rowman and Littlefield, I994, p. 8 I-IO8.

86. Je tiens à remercier Benoît Castelnérac ainsi qu'un commentateur anonyme pour leurs précieuses remarques qui m’ont permis d'améliorer cet article. 
Barnes, Jonathan. "Galen on Logic and Therapy " in Fridolf Kudlien and Richard J. Durling, eds., Galen's Method of Healing, Brill, I991, p. 50-I02.

Boudon-Millot, Véronique. "Art, science et conjecture chez Galien », dans J. Barnes et J. Jouanna, dir., Galien et la philosophie, Fondation Hardt, 2003, p. 269305 .

—. "Art, Science and Conjecture, From Hippocrates to Plato and Aristotle» in P. J. van der Eijk, ed., Hippocrates in Context, Brill, 2005, p. 89-99.

Chiaradonna, Riccardo. "Scienza e contingenza in Galeno", Conoscenza e contingenza nella tradizione aristotelica medievale, vol. 44, 2008, p. 9-28.

Di Piazza, Salvatore. "What Epistemological Status for Technai Stochastikai? Plato and Aristotle on Conjectural Knowledge ", Skepsis, 2007, p. I47-I 58.

—. "The Uncertainty of Medicine: Galen on the Notion of Techne Stochastike", Filozofia, vol. 71, 2016, p. 779-790.

Fortuna, Stefania. "La definizione della medicina in Galeno", La Parola del Passato, vol. 42, I987, p. I8I-I96.

—. "Il metodo della diagnosi in Galeno », Elenchos, vol. 22, 200I, p. 28I-304.

Frede, Micheal. "On Galen's Epistemology» in Vivian Nutton, ed., Galen: Problems and Prospects, The Wellcome Institute for History of Medicine, I98I, p. $65-86$.

- Essays in ancient philosophy, Minneapolis, University of Minnesota Press, I987, p. 382.

García-Ballester, Luis. "Galen as a Clinician: His Methods in Diagnosis ", Aufstieg und Niedergang der römischen, vol. 37, $\mathrm{n}^{\circ} 2$, I994, p. I636-I67I.

Gourevitch, Danielle. Le triangle hippocratique dans le monde gréco-romain. Le malade, sa maladie et son médecin, Rome, Écoles françaises d'Athènes et de Rome, I984, p. 569.

Hankinson, Robert J. "Galen on the Foundation of Science" in J. A. López Ferez, ed., Galeno: obra, pensamiento e influencia, I991, p. I 5-29.

- "Art and Experience: Greek Philosophy and the Status of Medicine", Quaestio, vol. 4, 2004, p. 3-24.

- dic., The Cambridge Companion to Galen, Cambridge University Press, 2008, p. 450 .

Hutchinson, Douglas S. "Doctrines of the Mean and the Debate Concerning Skills in Fourth-Century Medicine, Rhetoric and Ethics ", Apeiron, vol. 21, n ${ }^{\circ}$, I988, p. I7-52.

Ierodiakonou, Katerina. "Alexander of Aphrodisias on medicine as a stochastic art » in H.F.J. Horstmanshoff, Philip J. van der Eijk and P.H. Schrijvers, eds., Ancient medicine in its socio-cultural context vol. II, Rodopi, I995, p. 473485 .

Ingenkamp, Heinz Gerd. «Das $\sigma \tau o \chi \alpha ́ \sigma \alpha \sigma \theta \alpha \iota$ des Arztes» $(V M, \mathbb{S}$ 9), in François Lasserre et Philippe Mudry, dir., Formes de pensée dans la Collection hippocratique, Geneva, 1983, p. 257-262.

Inwood, Brad, "Goal and target in stoicism », The Journal of philosophy, 1986, vol. $83 \mathrm{n}^{\circ}$ Io, p. 547-556.

Jouanna, Jacques, et Véronique Boudon-Millot. «Présentation du projet d'édition de Galien dans la Collection des universités de France », Bulletin de l'Association Guillaume Budé, $\mathrm{n}^{\circ}$ 2, I993, p. IOI-I35. 
Lloyd, E. R. Geoffrey. Greek Science after Aristotle, New York, Norton, I973, p. I 89.

Mann, Joel E. «Prediction, Precision, and Practical Experience: the Hippocratics on Technē», Apeiron, vol. 4I, n 2, 2008, p. 89-I22.

Pietrobelli, Antoine. «Le modèle des démonstrations géométriques dans la médecine de Galien ", Bulletin de l'Association Guillaume Budé, vol. 2, 2009, p. I ıоI 30 .

Singer, P. N. "Levels of Explanation in Galen ", The Classical Quarterly, vol. 47, $\mathrm{n}^{\circ} 2$, 1997, p. 525-542.

Vegetti, Mario. "Modelli di medicina in Galeno ", in Vivian Nutton, dir., Galen: Problems and Prospects, Wellcome Institute of the History of Medicine, I98 I, p. 47-63.

__. "L'immagine del medico e lo statuto epistemologico della medicina in Galeno ", Aufstieg und Niedergang der römischen, vol. 37, $\mathrm{n}^{\circ}$ 2, I994, p. I672-I7I7. 